\title{
The caesarian section rate is rising
}

\author{
Attempts to curb it must be based on good obstetric care
}

Caesarean section is one of the oldest operations used in clinical practice. As clinical technology improved, caesarean deliveries became safer, and more commonly used. This procedure is now indicated in the management of a variety of obstetric settings.

The 20th century saw dramatic changes in the use of caesarean section. Initially, it was performed as a last resort in cases of obstructed labour, in patients with complications of the indication plus risks of the procedure. Older techniques of anaesthesia and other supporting facilities also contributed to the mortality and morbidity associated with caesarian section. Today its applications are much wider and include, for example, hypertension, fetal hypoxia and even salvageable congenital malformations. So the caesarean section rate had to rise.

In this issue of the CMJ Gunasekera, Wijesinghe and Goonewardena (pi47) present their views on caesarian section. The profession must take stock of the current practices, resources available and the need for caesarean section, so as to provide evidence-based guidelines for performing the operation. When formulating guidelines the logistics of providing the service in Sri Lanka must be taken into account.

The authors have considered the reasons for the rise in caesarean rate and dealt with its risks, benefits, and costs, and the practical considerations in providing the facility. The rise in caesarean rate is the result of sociodemographic changes, patient preferences and trends in medical practice. The total fertility rate (TFR) in Sri Lanka has enjoyed a healthy decline over the past three decades (1) reaching 2.26 in 1993 from 3.35 in 1974 (2). In the period 1987 (3) to 1993 (1) the TFR dropped by $20 \%$ from 2.82 to 2.26. During this period the decline in fertility also extended to women under the age of 30 years, whereas in previous years it was confined to those over 30 . This is a strong indicator of the wish to restrict family size to two children, and parents in Sri Lanka would naturally want to receive what they perceive as the best chance of success in these two pregnancies. An elective caesarean section, in their view, and perhaps also of the care provider, would be a way of achieving this.

The authors have quite rightly expressed their concern over definite (though diminishing) risks of caesarean delivery. The short term complications can and should be reduced by improving the quality of care. The previous caesarean scar would, however, remain as a serious problem in subsequent pregnancies. Statistics suggest that the success of a trial of labour in achieving a normal delivery when a past caesarean scar is present is high. A prospective study conducted over 12 months found that $63.2 \%$ of 68 cases selected for trial of labour with a previous caesarean scar had spontaneous or assisted vaginal deliveries (4). In contrast, an Australian multicentre study done over a S-year period in 11 major teaching hospitals showed that only $25.3 \%$ of the 21452 patients selected for trial of labour with a previous caesarean scar had a vaginal delivery (5). The former had (4) an incidence of scar dehiscence and rupture of $3.7 \%$, whereas the latter (5) had $0.3 \%$. The risk of rupture of a previous caesarean scar remains as a potential, unpredictable and sudden catastrophe in individual patients. Both studies have identified variables that predict good outcome in clinical practice. A greater use of trial of labour for a previous caesarean scar is to be encouraged, but the correct cases must be selected, and management should be undertaken at an institution capable of handling possible complications. Reduction in the caesarean rate would then occur in parallel with an improvement in the care provided. Of practical importance then is the question of how safe it would be for such women in Sri Lanka, particularly those from areas with inadequate access to specialist care, to face sudden onset of labour.

It is clear that the decision to perform that first caesarean section should be taken after deliberation and managed with a high level of operative skill. Specialist participation in the decision to perform a caesarean operation was only in $37.5 \%$ of cases (6). This is a cause for serious concern as decisions on the mode of delivery, particularly in an emergency, require the participation of clinicians with a high level of experience and expertise. This would improve the quality of care and help to reduce the caesarean section rate.

There is a steady rise in the incidence of pregnancies in women with chronic medical disorders such as congenital and acquired cardiac disease, diabetes, chronic renal disease, liver disorders and HIV infection. Medical disorders complicating pregnancy are now a leading cause of maternal death in Sri Lanka (7). In some of these conditions caesarean delivery would be clearly indicated. We have authoritative guidelines for scheduled caesarean delivery for the prevention of vertical transmission of HIV infection to the baby (8).

Although natural parturition may be the biological intention of most women, requests for elective caesarean delivery are on the increase. Gunasekera and colleagues have also discussed some of the ethical and moral issues involved. They compare the requests for caesarian section to the choice of the patient in deciding on management of non-life threatening interventions such as selecting a contraceptive and the treatment of menorrhagia. Caesarean section on demand appears to be prominent in the facilities provided by some units in the developed countries (9). In 
Sri Lanka the lack of patient involvement in decisionmaking for caesarian section has been addressed before (10). Whether it be for selection of a contraceptive, treatment for a neoplasm or choosing the mode of delivery by caesarian section in preference to the vaginal route, what is important is that the client has the right to make an "informed choice". This is in sharp contrast to the concept of "demanding" a method of treatment. The clinician has a tremendous responsibility to ensure that the patient (and her husband) selects the mode of delivery after being made aware of all relevant details, which includes the possible the effects on their future general and reproductive health. As stated by Gunasekera and colleagues, the obstetrician should not become a mere "technician" carrying out the wishes of the patient. What is required is a true partnership between the patient, her spouse and the care provider to ensure that the best results are obtained.

What is the significance of a rising caesarean rate? Is it necessary that rate of an individual unit should be below a specified cutoff level? In providing for caesarean delivery the need is to perform it when it is indicated while ensuring quality of care. The Annual Health Bulletin 1999 has reported on the place and type of delivery (11). Of the 43508 caesarean deliveries performed in the country in that year 290 (1.3\%) were in District Hospitals and Peripheral Units. Under most circumstances these would be considered very unsatisfactory venues for major operative delivery. There is a serious need to look at all aspects of maternity service delivery, and not only the rate of caesarean section.The article by Gunesekera, Wijesinghe and Goonewardena should be considered seriously, and guidelines formulated soon to provide the best possible obstetric service using the facilities and resources the country can afford.

\section{References}

1. Sri Lanka Demographic and Health Survey. Department of Census and Statistics, Ministry of Policy Planning and Implementation in Collaboration with Ministry of Health and Women's affairs, 1993.

2. World Fertility Survey, Sri Lanka. Department of Census and Statistics, 1978.

3. Sri Lanka Demographic and Health Survey. Department of Census and Statistics Printing Division, 1988.

4. Pathania K, Premi HK, Gupta T, Sood A. Delivery following previous caesarean section (a prospective study). Journal of Obstetrics and Gynaecology of India. 2000; SO: 63-7.

5. Appleton B, Targett C, Rasmussen M, Readman E, Sale F, Permzel $M$ and the VBAC study group. Vaginal birth after caesarean section: an Australian multicentre study. Australia and New Zealand Journal of Obstetrics and Gynaecology 2000; 40: 87-91.

6. Sirisena J, Jiffry N. Preventability of caesarean operations. Proceedings of the Kandy Society of Medicine 1994; 16: 45-6( abstract).

7. Annual Report on Family Health - 1997. Evaluation Unit, Family Health Bureau, Colombo, 1998; FH 10: p8.

8. American College of Obstetricians and Gynecologists Committee on Obstetric Practice; Committee opinion number 234. Scheduled caesarean delivery and the prevention of vertical transmission of HIV infection. International Journal of Gynaecology and Obstetrics 2001; 73: 279-81.

9. Jackson NV, Irvine LM. The influence of maternal request on the elective caesarean section rate. Journal of Obstetrics and Gynaecology 1998; 18: 115-9.

10. Randeniya C. Women's involvement with the decisions preceding their caesarean section and their degree of satisfaction. Workshop on presentation of research findings in Reproductive Health, 1997.

11. Annual Health Bulletin. Population Information Centre, Department of Health Services of Sri Lanka, 1999.

Harshalal R Seneviratne, Professor and Head, Department of Obstetrics and Gynaecology, Faculty of Medicine, University of Colombo.

\section{Cetirizine}

\section{Serious liver disorders underlying non-specific symptoms}

Serious liver disorders occurred in a 9-year old girl who was receiving certirizine ('Zyrlex') for allergic rhino-conjunctivitis with pruritus. During the 6-12 months after starting certirizine $10 \mathrm{mg} / \mathrm{day}$, the girl developed abdominal pain and episodes of nausea and vomiting. She was admitted to a children's clinic 18 months after starting cetirizine. Her AST and ALT levels were elevated at $7.24 \mathrm{pkat} / \mathrm{L}$ and $14.4 \mathrm{pkat} / \mathrm{L}$, respectively, and her lactate dehydrogenase level was elevated at $11 \mathrm{pkat} /$ L. Treatment with cetirizine was discontinued. Within 1 week, the girl's elevated liver enzyme levels had decreased by about 50\%, and they continued to improve over the next 6 weeks. At follow up, she had completely recovered.

The reported case emphasises the importance of following up non-specific symptoms in patients, which can sometimes hide a serious adverse drug reaction.

Reports in WHO-file: SCOT increased 12, SGPT increased 13, hepatic failure 1, hepatic necrosis 1

\section{Reference}

Medical Products Agency: 43, Apr 2001. 